\title{
A large spin-up rate measured with INTEGRAL in the high mass X-ray binary pulsar SAX J2103.5+4545*
}

\author{
L. Sidoli ${ }^{1}$, S. Mereghetti ${ }^{1}$, S. Larsson ${ }^{2}$, M. Chernyakova ${ }^{3}$, I. Kreykenbohm ${ }^{4,3}$, P. Kretschmar ${ }^{5,3}$, A. Paizis ${ }^{1,3}$, \\ A. Santangelo ${ }^{4}$, C. Ferrigno ${ }^{6}$, and M. Falanga ${ }^{7}$ \\ ${ }^{1}$ Istituto di Astrofisica Spaziale e Fisica Cosmica - Sezione di Milano - IASF/INAF, 20133 Milano, Italy \\ e-mail: sidoli@mi.iasf.cnr.it \\ 2 Department of Astronomy, Stockholm University, 10691 Stockholm, Sweden \\ 3 INTEGRAL Science Data Center, 1290 Versoix, Switzerland \\ ${ }^{4}$ Institut für Astronomie und Astrophysik - Astronomie, Univ. of Tübingen, 72076 Tübingen, Germany \\ ${ }^{5}$ ESA - European Space Astronomy Center, 28080 Villafranca del Castillo, Spain \\ ${ }^{6}$ Istituto di Astrofisica Spaziale e Fisica Cosmica - Sezione di Palermo - IASF/INAF, 90146 Palermo, Italy \\ 7 CEA Saclay, DSM/DAPNIA/Service d'Astrophysique (CNRS FRE 2591), 91191 Gif-sur-Yvette, France
}

Received 1 March 2005 / Accepted 20 May 2005

\begin{abstract}
The High Mass X-ray Binary Pulsar SAX J2103.5+4545 has been observed with INTEGRAL several times during the last outburst in 2002-2004. We report a comprehensive study of all INTEGRAL observations, allowing a study of the pulse period evolution during the recent outburst. We measured a very rapid spin-up episode, lasting $\sim 130$ days, which decreased the pulse period by $\sim 1.8 \mathrm{~s}$. The spin-up rate, $\dot{P} \sim-1.5 \times 10^{-7} \mathrm{~s} \mathrm{~s}^{-1}$, is the largest ever measured for SAX J2103.5+4545, and it is among the fastest for an accreting pulsar. The pulse profile shows evidence for temporal variability, apparently not related to the source flux or to the orbital phase. The X-ray spectrum is hard and there is significant emission up to $150 \mathrm{keV}$. A new derivation of the orbital period, based on RXTE data, is also reported.
\end{abstract}

Key words. X-rays: individuals: SAX J2103.5+4545 - X-rays: binaries - stars: neutron - accretion, accretion disks

\section{Introduction}

SAX J2103.5+4545 is a transient Be High Mass X-ray Binary (HMXRB) pulsar (pulse period $\sim 358 \mathrm{~s}$ ) discovered with the Wide Field Cameras (WFC) on-board BeppoSAX during an outburst in February-September 1997 (Hulleman et al. 1998). A peak intensity of $20 \mathrm{mCrab}$ was reached on 1997 April 11. The X-ray spectrum in the $2-25 \mathrm{keV}$ energy band was fit by a power-law with a photon index $\Gamma=1.27 \pm 0.14$ modified by photoelectric absorption at lower energies $\left(N_{\mathrm{H}}=3.1 \times\right.$ $10^{22} \mathrm{~cm}^{-2}$ ).

An orbital period of 12.68 days and an orbital eccentricity, $e$, of $0.4 \pm 0.2$ were measured with the Rossi X-ray Timing Explorer (RXTE) during a second outburst lasting about 14 months observed in 1999 (Baykal et al. 2000).

The likely optical counterpart is a B0Ve star $(V=14.2$; Reig \& Mavromatakis 2003; Reig et al. 2004), making SAX J2103.5+4545 the Be/X-ray binary with the shortest orbital period known. SAX J2103.5+4545 does not follow the

^ Based on observations with INTEGRAL, an ESA project with instruments and science data centre funded by ESA member states (especially the PI countries: Denmark, France, Germany, Italy, Switzerland, Spain), Czech Republic and Poland, and with participation of Russia and the USA. correlation found by Corbet (1986) between the orbital and spin periods in Be/X-ray binaries (which would imply an orbital period $\sim 190$ days).

The absorption in the direction of the optical counterpart indicates a distance of $6.5 \mathrm{kpc}$ (Reig et al. 2004), doubling the previous estimate of $\sim 3.2 \mathrm{kpc}$ (Baykal et al. 2002) based on a model for the spin-up produced by accretion torques (Ghosh \& Lamb 1979).

A transition from the spin-up phase to the spin-down regime was observed with RXTE during the decline in the X-ray flux of the 1999 outburst (Baykal et al. 2002). During the initial part of the outburst the source exhibited a spin-up phase (the pulse period decreased by $\sim 0.9 \mathrm{~s}$ in 150 days), but when the flux dropped the pulse frequency saturated and, as the flux continued to decline, a weak spin-down phase started. This correlation between spin-up rate and X-ray flux indicates the formation of an accretion disk during the periastron passage. The X-ray spectrum, described by a power-law with a cut-off around $8 \mathrm{keV}$, displayed an iron emission line at $6.4 \mathrm{keV}$ (Baykal et al. 2002).

During an XMM-Newton observation performed in January 2003, Inam et al. (2004), detected for the first time a soft blackbody component with a temperature of $1.9 \mathrm{keV}$ and radius $0.3 \mathrm{~km}$ (assuming a $3.2 \mathrm{kpc}$ distance), probably 
produced in the polar cap of the neutron star, as well as a transient quasi-periodic oscillation at $22.7 \mathrm{~s}$. Besides the blackbody component, the 1-20 keV spectrum (using XMM-Newton and RXTE data) was fit with a power-law with a cut-off at $7.89 \mathrm{keV}$ and an e-folding energy of $27.1 \mathrm{keV}$, together with an emission line at $6.42 \mathrm{keV}$. The equivalent width of this cold iron line was found to depend on the spin phase. Using RXTE data (MJD 52 611.48-MJD 52 668.90), Inam et al. (2004) derived a pulse period of $354.7940 \pm 0.0008 \mathrm{~s}$ (at the epoch of the XMM-Newton observation, MJD 52645.85 ) and a 57.5 day average spin-up rate of $(7.4 \pm 0.9) \times 10^{-13} \mathrm{~Hz} \mathrm{~s}^{-1}$.

Analyses of part of the INTEGRAL observations of the source have been reported by Lutovinov et al. (2003), Sidoli et al. (2004), Blay et al. (2004), Filippova et al. (2004) and Falanga et al. (2005). In this paper we report on the timing and spectral analysis of all INTEGRAL observations performed between December 2002 and July 2004.

\section{Observations}

The ESA INTEGRAL observatory, launched in October 2002, carries 3 co-aligned coded mask telescopes: the imager IBIS (Ubertini et al. 2003), which allows high-angular resolution imaging in the energy range $15 \mathrm{keV}-10 \mathrm{MeV}$, the spectrometer SPI (Vedrenne et al. 2003; $20 \mathrm{keV}-8 \mathrm{MeV}$ ) and the X-ray monitor JEM-X (Lund et al. 2003; 3-35 keV). IBIS is composed of a low-energy CdTe detector ISGRI (Lebrun et al. 2003), sensitive in the energy range from $15 \mathrm{keV}$ to $1 \mathrm{MeV}$, and a CsI detector (PICsIT; Labanti et al. 2003), designed for optimal performance at $511 \mathrm{keV}$, and sensitive in the $175 \mathrm{keV}-10 \mathrm{MeV}$ energy range.

The INTEGRAL satellite performs regular scans of the Galactic Plane (GPS) every 12 days as part of the Core Programme to monitor the timing and spectral properties of known X-ray sources, discover new transient sources and map the diffuse emission from the Galactic plane (Winkler et al. 2003).

From December 2002 to July 2004, SAX J2103.5+4545 was observed several times. Among these observations (170 pointings), some were performed during the satellite performance and verification phase (December 2002), during the GPS (January 2003-July 2004), or during guest observer observations now in the public archive (May 2003). We have performed a comprehensive study of all these observations (see Table 1), concentrating in particular on the timing properties of SAX J2103.5+4545.

The data reduction has been performed using the OSA 4.2 release of the INTEGRAL analysis software, with the corresponding response matrices. Each pointing has a typical exposure of $\sim 2-3 \mathrm{ks}$. Only observations where the SAX J2103.5+4545 position offset was less than $9^{\circ}$ have been considered. Only for a few of these pointings also JEM-X data were available, due to the smaller field of view of this instrument (offset less than $3.5^{\circ}$ ). SAX J2103.5+4545 has been detected in the energy range $20-40 \mathrm{keV}$ with IBIS/ISGRI in 113 pointings (see Table 1 ).

The 2-10 keV lightcurve of SAX J2103.5+4545 obtained with the RXTE All Sky Monitor (ASM) from 1997 to 2004 is
Table 1. Summary of the INTEGRAL observations of SAX J2103.5+4545. The observations have been grouped together for brevity. The 1st and 2nd columns list the Start and Stop time of each group of observations, Col. 3 reports the number of ISGRI pointings where the source is within $9^{\circ}$ of the pointing direction, Col. 4 reports the number of ISGRI/IBIS pointings where SAX J2103.5+4545 has been detected in the energy range $20-40 \mathrm{keV}$, while the last column shows if a search for periodicity has been succesfully carried out.

\begin{tabular}{llccc}
\hline \hline $\begin{array}{l}\text { Start time } \\
\text { (MJD) }\end{array}$ & $\begin{array}{l}\text { End time } \\
\text { (MJD) }\end{array}$ & $\begin{array}{c}\text { Number } \\
\text { of obs. }\end{array}$ & $\begin{array}{c}\text { Detections } \\
\text { Timing }\end{array}$ & \\
\hline 52617.86 & 52619.28 & 4 & 3 & $\mathrm{~N}$ \\
52620.74 & 52620.93 & 2 & 0 & $\mathrm{~N}$ \\
52629.63 & 52631.92 & 58 & 56 & $\mathrm{Y}$ \\
52636.66 & 52637.87 & 21 & 0 & $\mathrm{~N}$ \\
52638.46 & 52640.07 & 2 & 0 & $\mathrm{~N}$ \\
52653.32 & 52653.40 & 2 & 1 & $\mathrm{~N}$ \\
52722.85 & 52722.93 & 3 & 0 & $\mathrm{Y}$ \\
52737.03 & 52737.12 & 3 & 1 & $\mathrm{Y}$ \\
52746.10 & 52746.18 & 3 & 3 & $\mathrm{Y}$ \\
52761.26 & 52762.42 & 38 & 38 & $\mathrm{Y}$ \\
52770.05 & 52770.10 & 2 & 2 & $\mathrm{Y}$ \\
52782.06 & 52782.14 & 3 & 3 & $\mathrm{Y}$ \\
52835.9 & 52836.03 & 3 & 1 & $\mathrm{~N}$ \\
52985.41 & 52985.49 & 3 & 0 & $\mathrm{~N}$ \\
52994.38 & 52994.47 & 3 & 0 & $\mathrm{~N}$ \\
53019.37 & 53019.45 & 3 & 0 & $\mathrm{~N}$ \\
53045.61 & 53045.69 & 3 & 0 & $\mathrm{~N}$ \\
53090.96 & 53091.00 & 1 & 0 & $\mathrm{~N}$ \\
53102.48 & 53102.56 & 3 & 0 & $\mathrm{~N}$ \\
53114.16 & 53114.24 & 3 & 0 & $\mathrm{~N}$ \\
53126.09 & 53126.14 & 2 & 2 & $\mathrm{Y}$ \\
53137.88 & 53137.93 & 2 & 0 & $\mathrm{~N}$ \\
53164.97 & 53165.05 & 3 & 0 & $\mathrm{~N}$ \\
53188.96 & 53189.03 & 2 & 0 & $\mathrm{~N}$ \\
\hline
\end{tabular}

shown in Fig. 1. The times of the two previous outbursts observed with BeppoSAX (in 1997) and RXTE (in 1999), as well as the epoch of the INTEGRAL observations reported here, are indicated.

\section{Results}

\subsection{Timing analysis}

In order to perform the timing analysis, we selected events with a Pixel Illumination Function (PIF, pixel fraction illuminated by the source) larger than 0.5 . IBIS/ISGRI events have been collected in the energy band $20-40 \mathrm{keV}$, where the best statistics was available, while for JEM-X the range 3-30 keV has been used.

Arrival times have been corrected to the Solar System barycenter and for the Doppler delay due to the motion in the binary system. To perform this orbital correction, we adopted the ephemeris zero point from Baykal et al. (2000), 


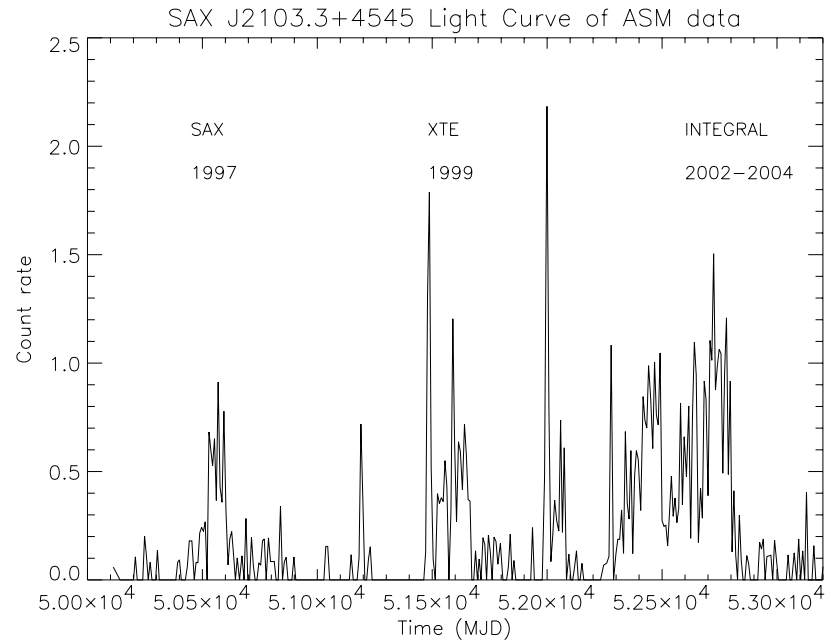

Fig. 1. ASM/RXTE lightcurve (8-days average) of SAX J2103.5+4545 from the outburst in 1997 to the end of August 2004. Three distinct outbursts are evident, and have been marked as "SAX 1997" (time of the source discovery with BeppoSAX, Hulleman et al. 1998), "XTE 1999" (observations with XTE reported by Baykal et al. 2000), "2002-2004" outburst observed with INTEGRAL.

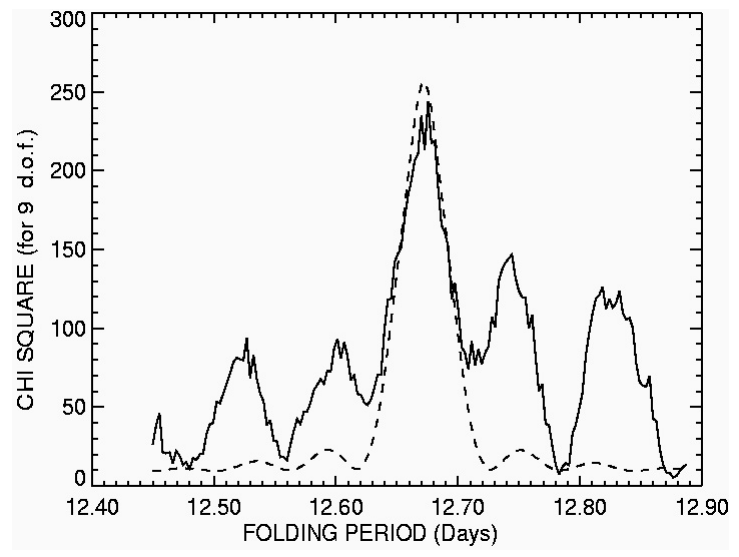

Fig. 2. $\chi^{2}$ distribution using all avilable RXTE-ASM data. It peaks at $12.670 \pm 0.005$ days, our improved determination of the orbital period for SAX J2103.5+4545. The solid line marks the data and the dashed line the fitted model (see text).

but used an updated orbital period, $P_{\text {orb }}=12.670 \pm 0.005$ days, that we calculated with epoch folding of all the available RXTE/ASM data (see Figs. 2 and 3). For our analysis, based on ASM dwell-by-dwell data, we used the epoch folding method described in Larsson (1996). The error estimate is based on Monte Carlo simulations and on the analysis of pulsations injected into the ASM lightcurve. From the Monte Carlo simulations we estimate the statistical error to be \pm 0.003 days. The slightly higher error estimate above includes also effect of the irregular source variability on the period determination. This latter effect is estimated from the injected pulsation.

Since the short duration of each single INTEGRAL pointing $(\sim 2-3 \mathrm{ks})$ leads to a large uncertainty in the determination of the pulse period (typically about $10 \mathrm{~s}$, even in presence of good statistics), we grouped the observations where a good estimate of the pulse period could be achieved in 7 separate

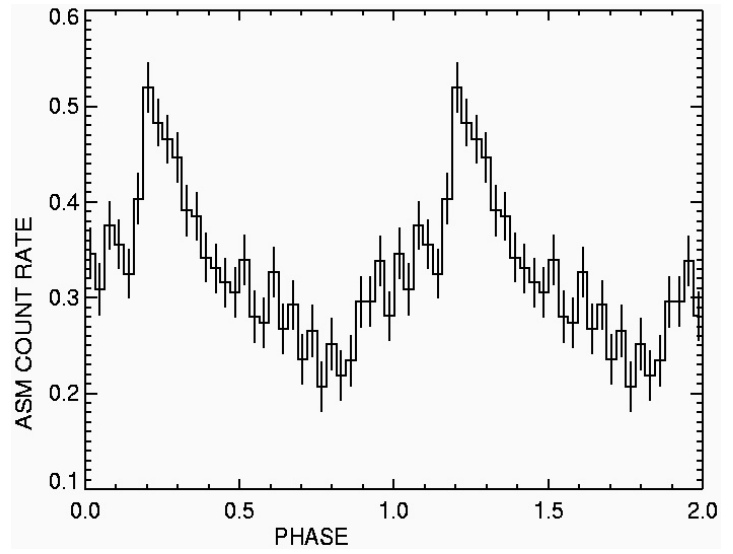

Fig. 3. SAX J2103.5+4545 RXTE-ASM lightcurve folded at the updated orbital period of $12.670 \pm 0.005$ days.

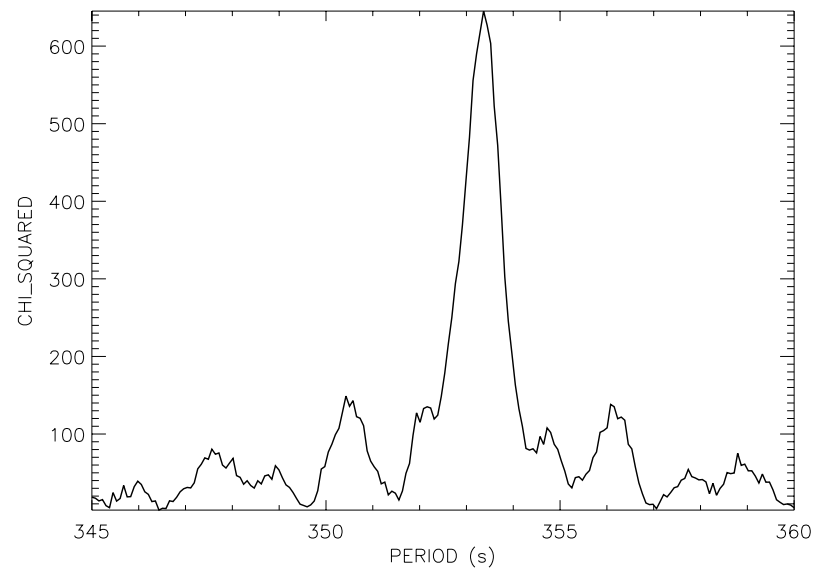

Fig. 4. Distribution of $\chi^{2}$ versus trial period for data set No. 4 (see Table 2) in the energy range $20-40 \mathrm{keV}$.

data sets consisting of the pointings performed during the same satellite revolution.

In Table 2 we list the start and end time of each group of observations, together with the source average IBIS/ISGRI count rate in the $20-40 \mathrm{keV}$ range. In data sets Nos. 1, 4 and 5 JEM-X data are also available, and have been used in the timing analysis.

We searched for the SAX J2103.5+4545 spin period by using epoch folding techniques: we produced $\chi^{2}$ distributions versus trial period, and then we fit them in order to derive the best-fit period for each data set, and its associated uncertainty (Leahy 1987). An example of a $\chi^{2}$ distribution is shown in Fig. 4.

The derived pulse periods are reported in Table 2. Our data sets Nos. 1 and 4 include observations partly reported by Blay et al. (2004), Filippova et al. (2004) and Falanga et al. (2004).

A period difference of $\sim 1.77 \mathrm{~s}$ in $\sim 130.5$ days is evident from the comparison of results from data sets Nos. 1 and 4 , which have the smallest uncertainty (Table 2). This spin-up is significantly higher than that measured during previous outbursts of this source.

The pulse period evolution during the INTEGRAL observations is shown in Fig. 7, where the period measured by Inam et al. (2004) with RXTE has been shown for completeness. 
Table 2. Results of the SAX J2103.5+4545 timing analysis. The 2nd and 3rd columns list the Start and Stop time of each data set, the 4th the averaged IBIS/ISGRI (20-40 keV) source count rate in these epochs, the 5th and 6th columns list the pulse periods measured in the seven INTEGRAL data sets. In data sets Nos. 2, 3, 6 and 7 the source was outside the JEM-X field of view. In data set No. 1 only the pulse period estimated with JEM-X is reported due to several telemetry gaps present in ISGRI lightcurve during this satellite revolution, which hampered a proper timing analysis.

\begin{tabular}{|c|c|c|c|c|c|}
\hline \multirow{2}{*}{$\begin{array}{c}\text { Data set } \\
\text { ID. }\end{array}$} & \multirow{2}{*}{$\begin{array}{l}\text { Start time } \\
(\mathrm{MJD})\end{array}$} & \multirow{2}{*}{$\begin{array}{l}\text { End time } \\
\text { (MJD) }\end{array}$} & \multirow{2}{*}{$\begin{array}{c}\text { IBIS/ISGRI } \\
\left(\mathrm{s}^{-1}\right)\end{array}$} & \multicolumn{2}{|c|}{ Pulse period (s) } \\
\hline & & & & IBIS/ISGRI & JEM-X \\
\hline 1 & 52629.91 & 52631.84 & $5.16 \pm 0.20$ & - & $355.09 \pm 0.03$ \\
\hline 2 & 52722.85 & 52722.90 & $4.28 \pm 0.18$ & 356. & - \\
\hline 3 & 52746.10 & 52746.18 & $6.07 \pm 0.25$ & $355.5 \pm 1.0$ & - \\
\hline 4 & 52761.26 & 52762.42 & $4.07 \pm 0.08$ & $353.32 \pm 0.03$ & $353.47 \pm 0.15$ \\
\hline 5 & 52770.05 & 52770.10 & $8.62 \pm 0.27$ & $351.8 \pm 1.3$ & $355.4 \pm 2.3$ \\
\hline 6 & 52782.06 & 52782.12 & $6.45 \pm 0.28$ & $351.3 \pm 2.3$ & - \\
\hline 7 & 53126.09 & 53126.14 & $4.34 \pm 0.28$ & 355. & - \\
\hline
\end{tabular}

A linear fit to all these data indicates an average spin-up with a rate of $\dot{P}=-(1.487 \pm 0.030) \times 10^{-7} \mathrm{~s} \mathrm{~s}^{-1}\left(\chi^{2}=21.3,8\right.$ degrees of freedom, d.o.f.).

A few examples of the folded light curves (background subtracted) obtained in different observations with ISGRI and JEM-X are shown in Figs. 5 and 6, respectively.

\subsection{Spectral analysis}

We used data from all INTEGRAL observations to derive a spectrum with a very high signal to noise ratio. Since the source exhibits no significant spectral evolution during the outburst, this can be safely done.

The average source spectrum from 5 to $200 \mathrm{keV}$, has been investigated by simultaneously fitting JEM-X (5-20 keV) together with IBIS/ISGRI (20-200 keV), and SPI (20-200 keV) spectra, achieving net exposure times of $40 \mathrm{ks}, 290 \mathrm{ks}$ and $130 \mathrm{ks}$, respectively for the three instruments. An additional uncertainty of $5 \%$ was added quadratically to the statistical errors to account for systematic uncertainties in instrumental responses. Free relative normalizations between the three instruments were included. Since the energy range $(5-200 \mathrm{keV})$ is not sensitive to the interstellar column density, we fixed it at $3 \times 10^{22} \mathrm{~cm}^{-2}$ (Hulleman et al. 1998).

A single power-law is not able to describe the broadband spectrum (reduced $\chi^{2}>2$ ). The best-fit $\left(\chi^{2}=121.9\right.$ for 129 d.o.f.) is an absorbed power-law with a high energy cut-off (see Fig. 8), with the following parameters: photon index $\Gamma=$ $1.53_{-0.07}^{+0.08}$, cut-off energy $E_{\mathrm{c}}=19_{-4}^{+11} \mathrm{keV}$ and e-folding energy $E_{\mathrm{f}}=32 \pm 3 \mathrm{keV}$. The $5-200 \mathrm{keV}$ observed flux is $\sim 1.05 \times$ $10^{-9} \mathrm{erg} \mathrm{cm}^{-2} \mathrm{~s}^{-1}$ (based on the JEM-X response matrix). The source flux in the $2-10 \mathrm{keV}$ energy range, corrected for the absorption, is $\sim 4.2 \times 10^{-10} \mathrm{erg} \mathrm{cm}^{-2} \mathrm{~s}^{-1}$, which translates into a luminosity $L_{\mathrm{X}}=2 \times 10^{36} \mathrm{erg} \mathrm{s}^{-1}(2-10 \mathrm{keV}$, for a distance of $6.5 \mathrm{kpc})$.

\section{Discussion}

We have studied all the available INTEGRAL observations of SAX J2103.5+4545, obtained from December 2002 to
July 2004. They cover part of the latest source outburst, which is the longest and brightest observed from this source since its discovery in 1997.

To perform a proper timing analysis of the INTEGRAL observations, we re-determined the orbital period of the binary system using ASM/XTE public data, obtaining a refined $P_{\text {orb }}$ of $12.670 \pm 0.005$ days (to be compared with $P_{\text {orb }}$ of $12.68 \pm$ 0.25 days, measured with XTE during the 1999 outburst by Baykal et al. 2000).

The SAX J2103.5+4545 pulse profiles observed with INTEGRAL show evidence for temporal variability over the same energy range (15-40 keV), where a single asymmetric pulse profile seems to evolve to a double-peaked profile in some of the observations. On the other hand, in the lightcurves extracted at softer energies $(3-30 \mathrm{keV})$ there is no clear evidence for a secondary peak. The INTEGRAL observations unfortunately do not cover the entire orbital phase; in particular, the data sets Nos. 3, 5, 6 and 7 sample roughly the same orbital phase (these are indeed GPS observations, which are performed every 12 days, a value close to the source orbital period). Thus, apparently the complex variability of the pulse profile does not depend on the orbital phase. Also an obvious dependence on the source luminosity seems to be unlikely, since we observe different pulse periods from observations at similar fluxes (e.g., data sets Nos. 2 and 7). The complex variability of the pulse profiles is a common feature among the class of accreting binary pulsars, also over the same energy range (White et al. 1983; Bildsten et al. 1997). In some sources the pulse shape variability shows a clear dependence on the source luminosity (first observed in EXO 2030+375, Parmar et al. 1989) or on the energy range (e.g. Vela X-1, Kretschmar et al. 1997). A simple geometric model with two pencil beams coming from the magnetic poles is too simple to explain the pulse profiles in most pulsars, and a more complex emission pattern or the inclusion of scattering effects in the transfer through highly magnetized plasmas are needed (e.g., Nagel 1981). A computation of pulse profiles of medium-luminosity $\left(L_{\mathrm{X}} \sim 10^{36} \mathrm{erg} \mathrm{s}^{-1}\right)$ binary X-ray pulsars has been performed by Kraus et al. (2003), who found that energy-dependent peaks in the profiles are mainly due to energy-dependent relative importance of the halo (which 

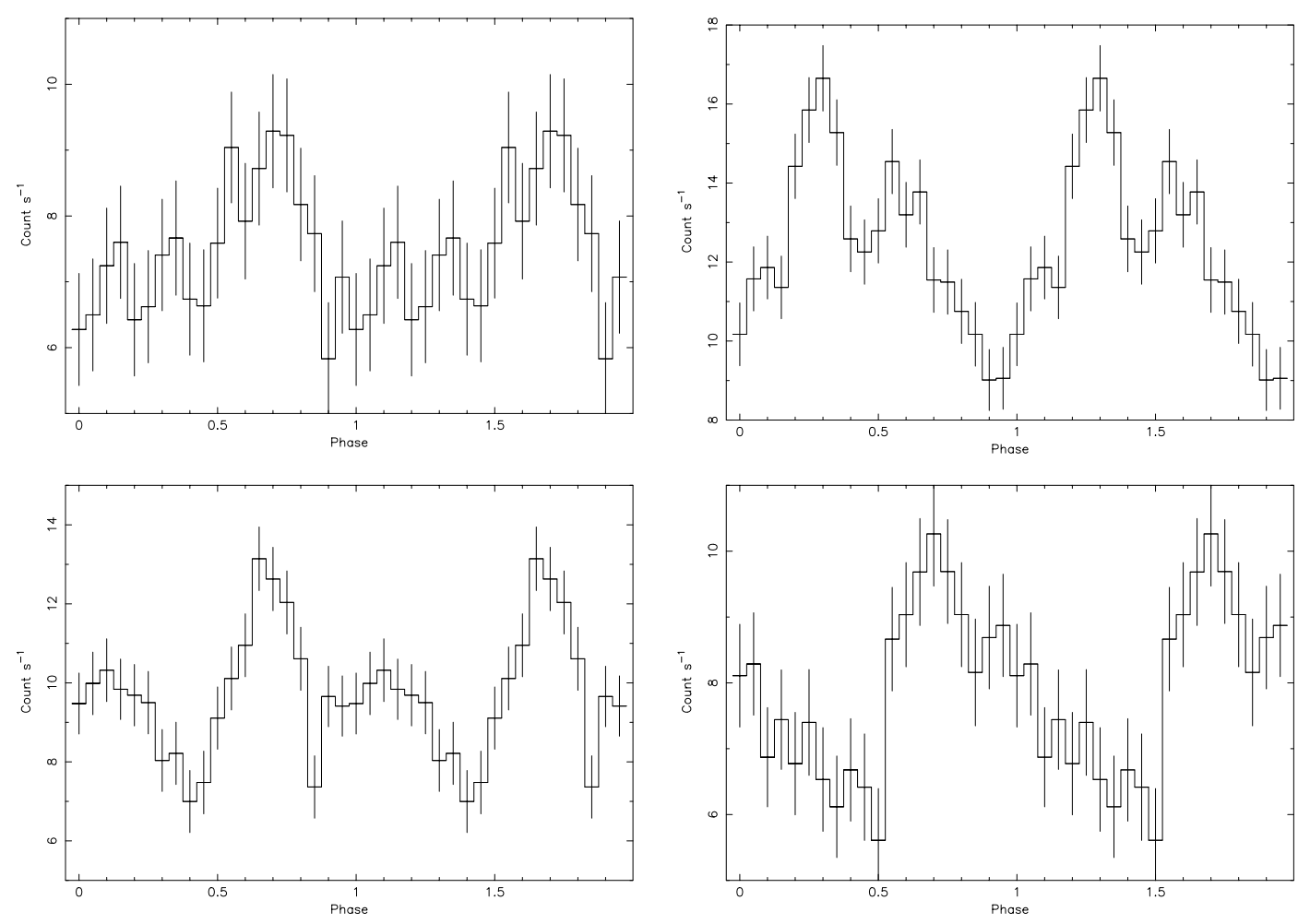

Fig. 5. Few examples of SAX J2103.5+4545 pulse profiles from INTEGRAL observations where a different shape is evident. From top to bottom, from the left to the right, profiles are from data sets Nos. 2, 5, 6 and 7, all in the energy range 15-40 keV. The zero phase is arbitrary. The pulsed fraction, defined as the ratio of maximum minus minimum to maximum, is $\sim 33 \%$ for data set No. 2 , while $\sim 47 \%-50 \%$ for the other three lightcurves.
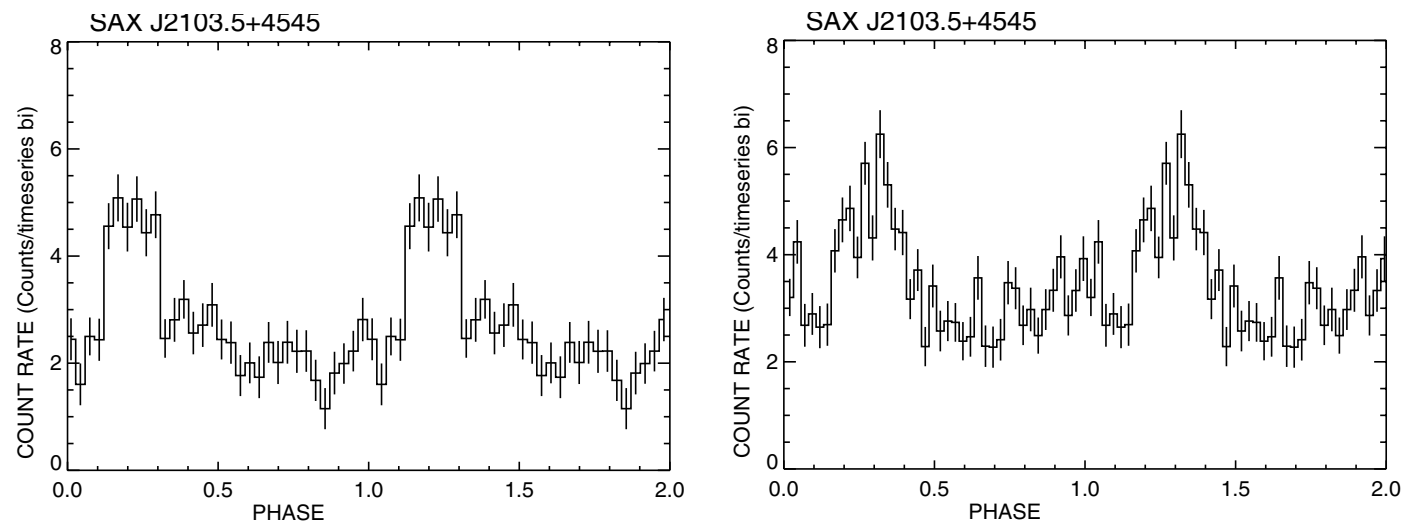

Fig. 6. Pulse profiles at soft energies (3-30 keV) with JEM-X, from data set No. 1 (left panel) and No. 4 (right panel).

forms around the accretion funnel where the neutron star surface is irradiated) and the column contributions to the observed flux. It is also possible that the temporal variability of the pulse profile we observe in the same energy range can be due to timedependent emission pattern, or to changes in the opacity of the magnetized plasma where the radiation propagates.

The pulse period evolution measured with INTEGRAL shows clear evidence for a spin-up episode, lasting at least $\sim 130$ days from MJD 52629.9 (data set No. 1) to MJD 52761.3 (data set No. 4), where the pulse period decreased by $\sim 1.77 \mathrm{~s}$.

Considering the overall pulse period history during the latest outburst (Fig. 7), a linear fit results in a spin-up rate $\dot{P}=$ $-(1.487 \pm 0.030) \times 10^{-7} \mathrm{~s} \mathrm{~s}^{-1}$.
We cannot exclude a more complex evolution of the pulse period than a simple steady spin-up over more than 1.3 years. In particular, it is likely that the spin-up trend induced by the accretion does not extend until the epoch of the data set No. 7 .

A variation in the accretion rate can modify the pulse period derivative. A correlation of the spin-up rate with the X-ray flux has been indeed found for SAX J2103.5+4545 by Baykal et al. (2002) during the 1999 outburst, as predicted by the Ghosh \& Lamb (1979) model for the torques exerted on the neutron star during the accretion through a disk. The existence of this correlation is indeed indicative of the formation of an accretion disk around SAX J2103.5+4545 during that outburst. The last pulse period measured with INTEGRAL (data set No. 7) has been determined after a long faint state lasting about 300 days (Fig. 7, 


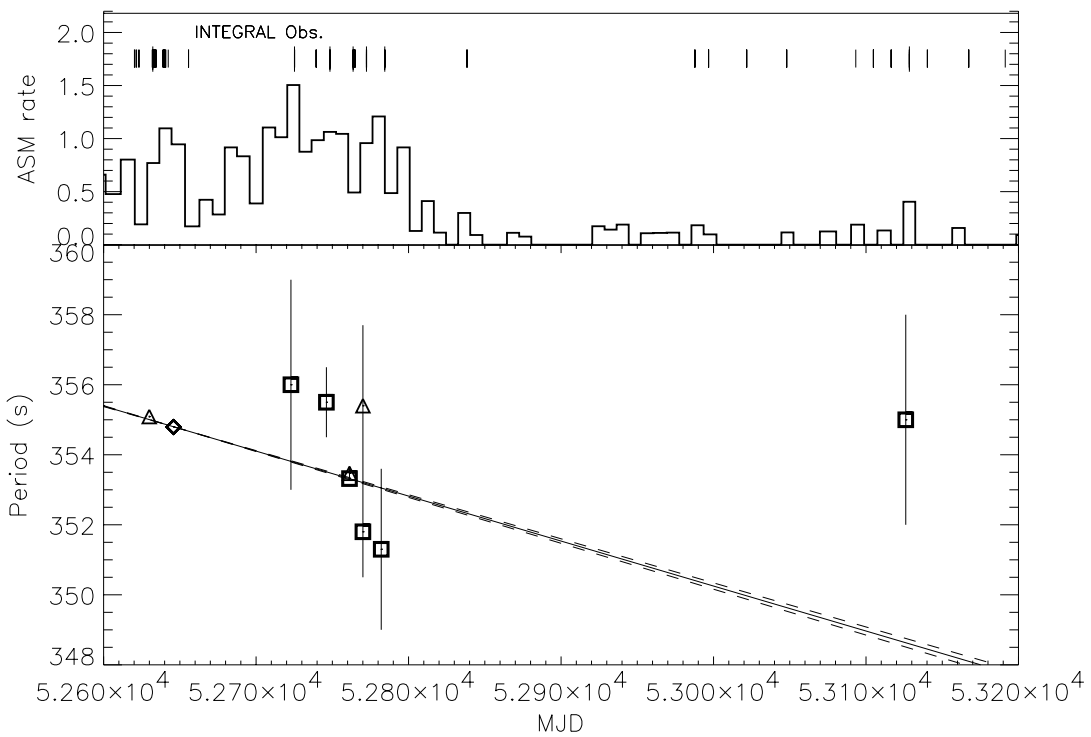

Fig. 7. Upper panel: a close-up view of the ASM/RXTE lightcurve (8-days average) during the INTEGRAL observations. The epochs of the INTEGRAL observations have been marked with vertical lines. Lower panel: SAX J2103.5+4545 pulse period evolution (triangles mark JEM-X results; squares IBIS/ISGRI results). The diamonds indicate the period measured with XTE by Inam et al. (2004). A linear fit to all the data is reported (solid line) together with the 1- $\sigma$ uncertainty (dashed lines).
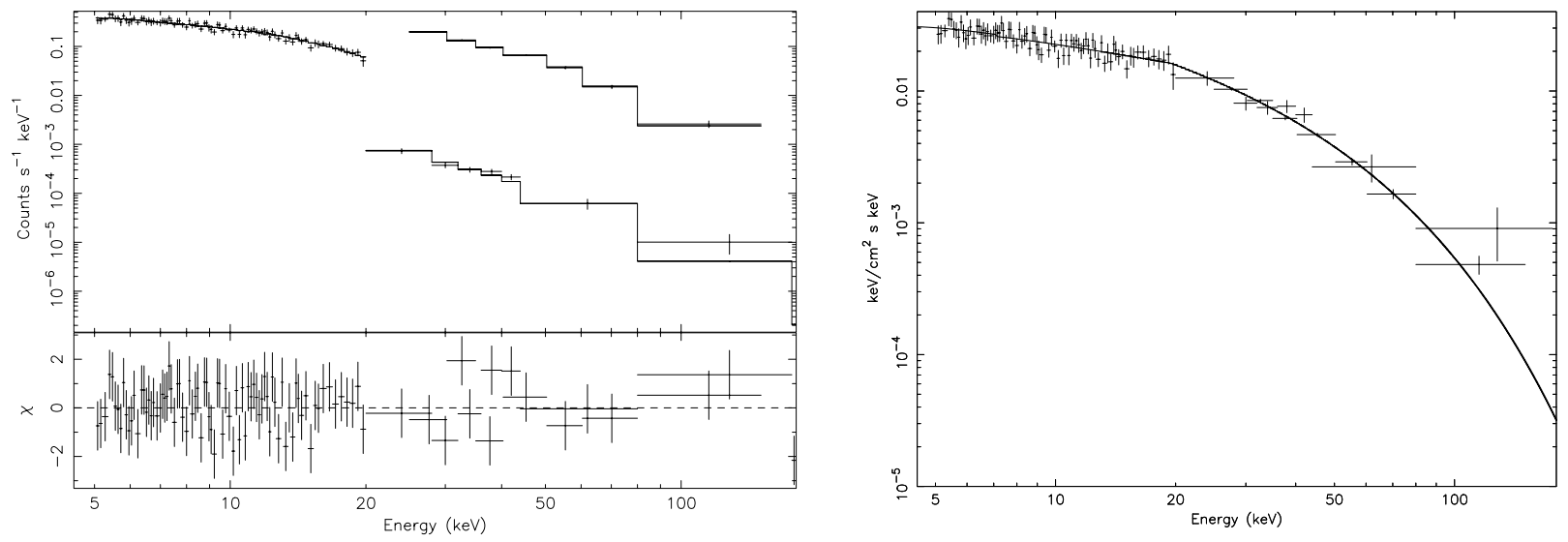

Fig. 8. Left panel: SAX J2103.5+4545 counts spectrum (5-200 keV) extracted from JEM-X (5-20 keV), IBIS/ISGRI (20-200 keV, upper counts spectrum) and SPI (20-200 keV, lower spectrum) instruments. The best-fit model (histogram) is an absorbed power-law with a cut-off at high energies (see text for the best-fit parameters). The residuals displayed in the lower panel are in units of standard deviations. Right panel: SAX J2103.5+4545 unfolded spectrum together with the best-fit model (solid line).

upper panel). The reduced accretion rate could have increased the SAX J2103.5+4545 pulse period (a spin-down phase followed the spin-up trend during the 1999 SAX J2103.5+4545 outburst, Baykal et al. 2002), although the large uncertainty in the pulse period at that epoch $(P=355 \pm 3 \mathrm{~s})$ does not permit to draw firm conclusions.

The formation of an accretion disk in SAX J2103.5+4545 during the outburst in 1999 has been established from RXTE data (Baykal et al. 2002). Furthermore, the fact that our estimate of the spin-up rate (the highest ever measured in this source) has been measured during the brightest outburst observed from SAX J2103.5+4545 indicates that the correlation between luminosity and spin-up is still valid. It is thus tempting to discuss our results on the source spin-up rate in the framework of the Ghosh \& Lamb (1979) theory.
In Fig. 9 we compare the measured pulse period derivative of $\sim-1.49 \times 10^{-7} \mathrm{~s} \mathrm{~s}^{-1}$ with the theoretical relation between the spin-up rate of a $1.4 M_{\odot}$ neutron star and the quantity $P L^{3 / 7}$, where $P$ is the pulse period in seconds, and $L$ is the source luminosity in units of $10^{37} \mathrm{erg} \mathrm{s}^{-1}$ (Ghosh \& Lamb 1979). Thus, assuming that the distance of $6.5 \mathrm{kpc}$ is correct, a magnetic moment of $\sim 1.6 \times 10^{30}$ Gauss $\mathrm{cm}^{3}$ is needed to explain the spin-up rate. This translates into a magnetic field of $\sim 10^{12}$ Gauss, which would imply a cyclotron line at energies around $\sim 10-20 \mathrm{keV}$, not observed in the source spectrum. The absence of lines could suggest a much higher neutron star magnetic field, $\sim 10^{13}$ Gauss, but this would imply a smaller distance, around $5 \mathrm{kpc}$. A distance of $5 \mathrm{kpc}$ also agrees with the maximum spin-up trend measured with XTE during the 1999 outburst (Baykal et al. 2002): a pulse period derivative 


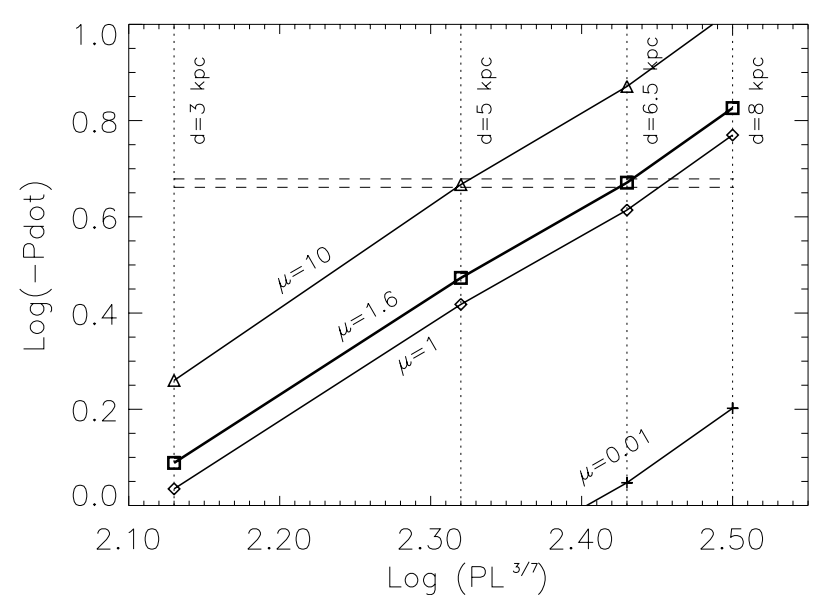

Fig. 9. Measured spin-up rate (horizontal dashed lines show the $1 \sigma$ uncertainty interval for $\left.\dot{P}=-(1.487 \pm 0.030) \times 10^{-7} \mathrm{~s} \mathrm{~s}^{-1}\right)$ compared with the theoretical relation (from Ghosh \& Lamb 1979) between the spin-up rate $-\dot{P}$ (here in units of $\mathrm{yr} \mathrm{s}^{-1}$ ) and the quantity $P L^{3 / 7}$, where $P$ is the pulse period in seconds, and $L$ is the source luminosity in units of $10^{37} \mathrm{erg} \mathrm{s}^{-1}$. Each curve has been calculated for a different value of the magnetic moment $\mu$ (in units of $10^{30}$ Gauss $\mathrm{cm}^{3}$; triangles

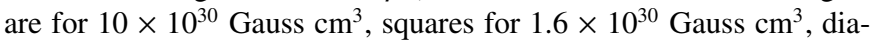
monds for $10^{30}$ Gauss $\mathrm{cm}^{3}$, and crosses for $0.01 \times 10^{30} \mathrm{Gauss}^{3}$ ). A $1.4 M_{\odot}$ neutron star, a pulse period $P=353.32 \mathrm{~s}$ and a source flux of $10^{-9} \mathrm{erg} \mathrm{cm}^{-2} \mathrm{~s}^{-1}$ have been considered. Vertical dotted lines show loci of equal source distance d: from left to right, $d=3 \mathrm{kpc}, d=5 \mathrm{kpc}$, $d=6.5 \mathrm{kpc}, d=8 \mathrm{kpc}$.

of $\sim 8.9 \times 10^{-8} \mathrm{~s} \mathrm{~s}^{-1}$ (and pulse period $P=358.166 \mathrm{~s}$ ) for a source flux of $\sim 6 \times 10^{-10} \mathrm{erg} \mathrm{cm}^{-2} \mathrm{~s}^{-1}$ can be explained with a neutron star of $1.4 M_{\odot}$ and a magnetic moment $\sim 10^{31}$ Gauss $\mathrm{cm}^{3}$, located at a distance of about $5 \mathrm{kpc}$.

However, note that there are uncertainties in the dimensionless angular momentum adopted in the accretion torque theory (Li \& Wang 1996), which could significantly modify the estimate of the source distance derived with this method (see, e.g., Tsygankov \& Lutovinov 2005, on the binary pulsar KS 1947+300). Another uncertainty is due to the source flux we adopted here, which is not the bolometric flux and has been measured from the average INTEGRAL spectrum in the energy range 5-200 keV.

The spin-up rate measured with INTEGRAL from SAX J2103.5+4545 is the largest experienced by this source (likely because of the higher brightness of the latest outburst) and is among the largest found for an X-ray binary pulsar. The frequent monitoring (as that performed with INTEGRAL during the GPS) of accreting pulsars is essential in the determination of the pulse period evolution, and crucial to test accretion theories.

Acknowledgements. L.S. thanks Joern Wilms for useful suggestions. We acknowledge the Italian Space Agency financial and programmatic support via contract I/R/046/04.

\section{References}

Baykal, A., Stark, M. J., \& Swank, J. H. 2000, ApJ, 544, L129

Baykal, A., Stark, M. J., \& Swank, J. H. 2002, ApJ, 569, 903

Bildsten, L., Chakrabarty, D., Chiu, J., et al. 1997, ApJS, 113, 367

Blay, P., Reig, P., Martinez Nunez, S., et al. 2004, A\&A, 427, 293

Corbet, R. H. D. 1986, MNRAS, 220, 1047

Filippova, E. V., Lutovinov, A. A., Shtykovsky, P. E., et al. 2004, Astron. Lett., 30, 824 [arXiv: astro-ph/0410590]

Falanga, M., Di Salvo, T., Burderi, L., et al. 2005, A\&A, 436, 313

Ghosh, P., \& Lamb, F. K. 1979, ApJ, 234, 296

Hulleman, F., in't Zand, J. J. M., \& Heise, J. 1998, A\&A, 337, L25

Inam, S. C., Baykal, A., Swank, J., et al. 2004, ApJ, 616, 463

Kraus, U., Zahn, C., Weth, C., et al. 2003, ApJ, 590, 424

Kretschmar, P., Pan, H. C., Kendziorra, E., et al. 1997, A\&A, 325, 623

Labanti, C., Di Cocco, G., Ferro, G., et al. 2003, A\&A, 411, L149

Larsson, S. 1996, A\&AS, 117, 197

Leahy, D. A. 1987, A\&A, 180, 275

Lebrun, F., Leray, J. P., Lavocat, P., et al. 2003, A\&A, 411, L141

Li, X.-D., \& Wang, Z.-R. 1996, A\&A, 307, L5

Lund, N., Brandt, S., Budtz-Jorgensen, C., et al. 2003, A\&A, 411, L231

Lutovinov, A. A., Molkov, S. V., \& Revnivtsev, M. G. 2003, Astron. Lett., 29, 713

Nagel, W. 1981, ApJ, 251, 278

Parmar, A. N., White, N. E., \& Stella, L. 1989, ApJ, 338, 373

Reig, P., \& Mavromatakis, F. 2003, Atel, 173

Reig, P., Negueruela, I., Fabregat, J., et al. 2004, A\&A, 421, 673

Sidoli, L., Mereghetti, S., Larsson, S., et al. 2004, Proc of the V INTEGRAL Workshop, SP-552, 475

Tsygankov, S. S., \& Lutovinov, A. A. 2005, Astron. Lett., 31, 88

Ubertini, P., Lebrun, F., Di Cocco, G., et al. 2003, A\&A, 411, L131

Vedrenne, G., Roques, J.-P., Schoenfelder, V., et al. 2003, A\&A, 411, L63

White, N. E., Swank, J. H., \& Holt, S. S. 1983, ApJ, 270, 711

Winkler, C., Gehrels, N., Schoenfelder, V., et al. 2003, A\&A, 411, L349 